\title{
Recommendations for the rehabilitation and release of wild-born, captive-raised cheetahs: the importance of pre- and post-release management for optimizing survival
}

Eli H. Walker, Stijn Verschueren

AnNe SChMidT-KÜNTZEL and Laurie Marker

\begin{abstract}
Large carnivores are frequently released for conservation purposes, but early efforts struggled with inadequate monitoring and reporting, resulting in poor understanding of success. Although managers have improved release practice and monitoring, the use of orphaned, captive-raised large carnivores for release remains controversial because of the potential influence of captivity and the possible lack of natural behaviours in such individuals. Yet, rehabilitating orphaned individuals for release could help mitigate pressures on vulnerable wild populations. We present a case study on the rearing, rehabilitation and release of wild-born cheetahs Acinonyx jubatus that were rescued as orphans in Namibia. Our aim was to develop a methodological framework for cheetah release planning and post-release management based on the outcome of release trials conducted during 2004-2018. Between 2001 and 2012, we rescued 86 orphaned cheetahs from the wild. Of these, $36(42 \%)$ were selected as release candidates. We found high success rates $(75-96 \%)$ of selected individuals in achieving independence post-release. Additionally, annual survival estimates for rehabilitated individuals that reached independence were comparable to those of wild counterparts described by other studies, and some rehabilitated individuals reproduced with wild conspecifics. Our findings demonstrate the ability of wild-born, captive-raised cheetahs to transition back into the wild with strategic pre- and post-release management directed towards optimizing survival. This includes selecting appropriate release candidates, forming artificial coalitions, balancing habituation levels during captivity, choosing appropriate release sites, and providing strategic support during post-release monitoring. We encourage scientists and managers to implement and refine our protocol for rehabilitation throughout the cheetah's current and historic range.
\end{abstract}

Keywords Captive-raised, cheetah, large carnivore, postrelease monitoring, rehabilitation, reintroduction, release, translocation

ELI H. WALKER (Corresponding author, (10) orcid.org/0000-0003-4946-7122), Stijn Verschueren (®D orcid.org/0000-0003-1420-7689), AnNe SChMidt-KüNTZEL (D) orcid.org/0000-0001-5108-2606) and LAURIE MARKER (미 orcid.org/00000002-1636-2191, director@cheetah.org) Cheetah Conservation Fund, PO Box 1755, Otjiwarongo, Namibia

Received 12 June 2020. Revision requested 6 August 2020.

Accepted 5 February 2021. First published online 15 February 2022.
Supplementary material for this article is available at doi.org/10.1017/So030605321000235

\section{Introduction}

The global decline in cheetah Acinonyx jubatus popu1 lations has led to their current IUCN Red List categorization as Vulnerable in most of Africa and Critically Endangered in north-west Africa and Iran (Durant et al., 2015). Namibia is a stronghold for the cheetah and possibly holds $>25 \%$ of the estimated global cheetah population (Marker et al., 2018a,b) of 7,100 adult and adolescent individuals (Durant et al., 2015). In Namibia, 90\% of the cheetah population lives outside protected areas and occupies livestock and game farmlands, resulting in a high risk of loss as a result of human-wildlife conflict (Morsbach, 1987; Marker-Kraus et al., 1996; Marker et al., 2007, 2018a,b). The Cheetah Conservation Fund rescues cheetahs throughout Namibia orphaned as a result of human-wildlife conflict and, where possible, helps rescued cheetahs transition back into the wild.

The release of cheetahs and other large carnivores for conservation purposes (rehabilitation, reintroduction and reinforcement translocation, hereafter referred to as 'release') is not a new practice and numerous releases have occurred across Africa (Hayward et al., 2007; Marnewick et al., 2009; Houser et al., 2011; Weise et al., 2015; Boast et al., 2018). Previously, release efforts of cheetahs were hindered by issues with design and decision-making, lack of monitoring, and inadequate reporting (Boast et al., 2018). However, improved knowledge and expertise garnered from continual effort and improved pre- and post-release management has led to high levels of success (Marnewick et al., 2009; Buk et al., 2018; Vebber et al., 2020).

The release of captive-bred or captive-raised individuals presents an added layer of difficulty as such individuals have shown poor survival post-release (Jule et al., 2008; Hunter \& Rabinowitz, 2009). This has mainly been attributed to these animals lacking natural behaviours associated with survival, foraging success and reproductive fitness (Snyder et al., 1996; Vickery \& Mason, 2003). Yet, the few studies that documented successful cheetah rehabilitations found that post-release ranging and prey selection behaviour were 
similar to those of their wild counterparts (Houser et al., 2011; Vebber et al., 2020).

As wildlife populations continue to decline, the value of releasing captive-bred and captive-raised individuals may grow. By increasing post-release survival, use of these individuals for release efforts vs wild-caught individuals could mitigate the pressure on vulnerable and/or dwindling wild populations (Wilson \& Price, 1994; Schwartz et al., 2018). Cheetahs naturally live at low population densities and are at risk of further decline as a result of human-wildlife conflict, habitat loss and illegal trade (Marker et al., 2018a,b). Rehabilitation of orphaned/trapped individuals could therefore be a complementary approach for cheetah conservation.

Despite recent successful releases of cheetahs, the need for improved understanding and methodology, and for evidence-based decision-making and management in reintroduction practices for carnivores, is well established (Hayward, et al., 2007; Gusset et al., 2008; Jule et al., 2008; Taylor et al., 2017). Here we present a methodological framework for cheetah rehabilitation and post-release management based on the outcome of release trials conducted by the Cheetah Conservation Fund during 2004-2018. We present the characteristics of release sites and a detailed description of the selection process of release candidates, followed by an overview of our preand post-release management protocol. Then, we analyse post-release survival in light of the main biological (age when orphaned, sex), ecological (group size, release site) and management-related (captivity time, trainingrelease) factors that we expected to affect post-release survival. These findings are incorporated into our management recommendations for the rehabilitation and release of wild-born, captive-raised cheetahs, and we encourage scientists and managers to implement, expand and refine this protocol throughout the cheetah's current and historical range.

\section{Methods}

\section{Release site characteristics}

The study area included three release sites in Namibia within the cheetah's current and historical range (Table 1, Fig. 1, Supplementary Table 1). Assessed by surveys and expert knowledge, all sites had sufficient prey available, suitable cheetah habitat, and natural and artificial water points that provided water year-round. Release sites were sufficiently large to meet the ecological requirements of cheetahs (Lindsey et al., 2011). Competing carnivores were present in all release sites. Cheetahs may persist well in systems with dominant competitors through fine-scale avoidance strategies (Durant, 1998, 2000; Broekhuis et al.,
2013; Swanson et al., 2014, 2016), thus the presence of other carnivores was not considered to be a limiting factor. Resident conspecifics may negatively influence release efforts, but established, long-term monitoring was present in all release sites and none had large resident cheetah populations. In addition, both the NamibRand Nature Reserve and the Greater Waterberg Landscape facilitate natural regulation of existing cheetah populations through natural dispersal, as they are open systems. NamibRand Nature Reserve was established as an open system in 1984. The Greater Waterberg Landscape is an open system with mixed land use of livestock and game farming and includes the Bellebenno release training camp. Erindi Private Game Reserve is a private fenced reserve that was converted from a cattle farm in 2008.

\section{Release candidate selection}

The selection of cheetahs to be released was based on the following parameters:

Age at orphaning We considered individuals orphaned at an age of 6 months or older as release candidates. At this age, they have experienced 4 months or more out of the natal den with their mother, learning survival skills (Caro, 1994). This enabled us to minimize their habituation to humans during captivity, as they did not need the close care that younger orphans required. Individuals orphaned at 3-5 months were considered for release if bonded to older orphans that qualified. Once bonded, the older orphans help minimize habituation levels in the younger orphans.

Level of habituation We assessed the degree of habituation of each release candidate with the classification criteria described by Weise et al. (2015) (Table 2), adapted for this study. Individuals classified as habituated were not considered releasable. We aimed for candidates to be semihabituated at the time of release, to allow the monitoring team to track and observe the individual post-release reliably. This level of habituation could be maintained for most individuals orphaned at $\geq 6$ months of age (and their younger coalition members), as those candidates only had exposure to human activity during husbandry routines (e.g. feeding, health check, management training). By maintaining the optimal habituation level for all candidates, we aimed to reduce the impact of captivity time on survival probability (Weise et al., 2015).

Social grouping Adult female cheetahs live solitarily except when breeding or raising cubs, whereas males form lifelong groups with their brothers, known as coalitions (Caro, 1994; Wachter et al., 2018). Living in a coalition may improve male survival probability (Durant et al., 2004). If bonded at an 
TABLE 1 Summary information for the three sites in Namibia were cheetahs Acinonyx jubatus were released (Fig. 1).

\begin{tabular}{llll}
\hline Site name & Greater Waterberg Landscape & Erindi Private Game Reserve & NamibRand Nature Reserve \\
\hline Area $\left(\mathrm{km}^{2}\right)$ & 2,740 & 790 & 1,723 \\
Game fence & No & Yes & No \\
Land use & Farmland, reserve & Reserve & Reserve \\
Mean annual rainfall $(\mathrm{mm})$ & $400-500$ & $300-350$ & 70 \\
Annual temperature range $\left({ }^{\circ} \mathrm{C}\right)$ & $0-43$ & $3-43$ & $-4-43$ \\
Topography & Flat, with plateau & Flat to mountainous & Rocky hillsides, sandy plains \\
Vegetation type & Wooded, patches of open & Wooded plains, open savannah & Open savannah, desert with \\
& savannah & & vegetated dunes \\
Water & Artificial waterholes, & Natural \& artificial waterholes, & Artificial waterholes \\
& dry rivers & dry rivers & $123.4 \pm 133.6$ \\
Mean prey density $\pm \mathrm{SE}$ (per 1,000 ha) & $320.8 \pm 53.6$ & $191.3 \pm 3.4$ &
\end{tabular}

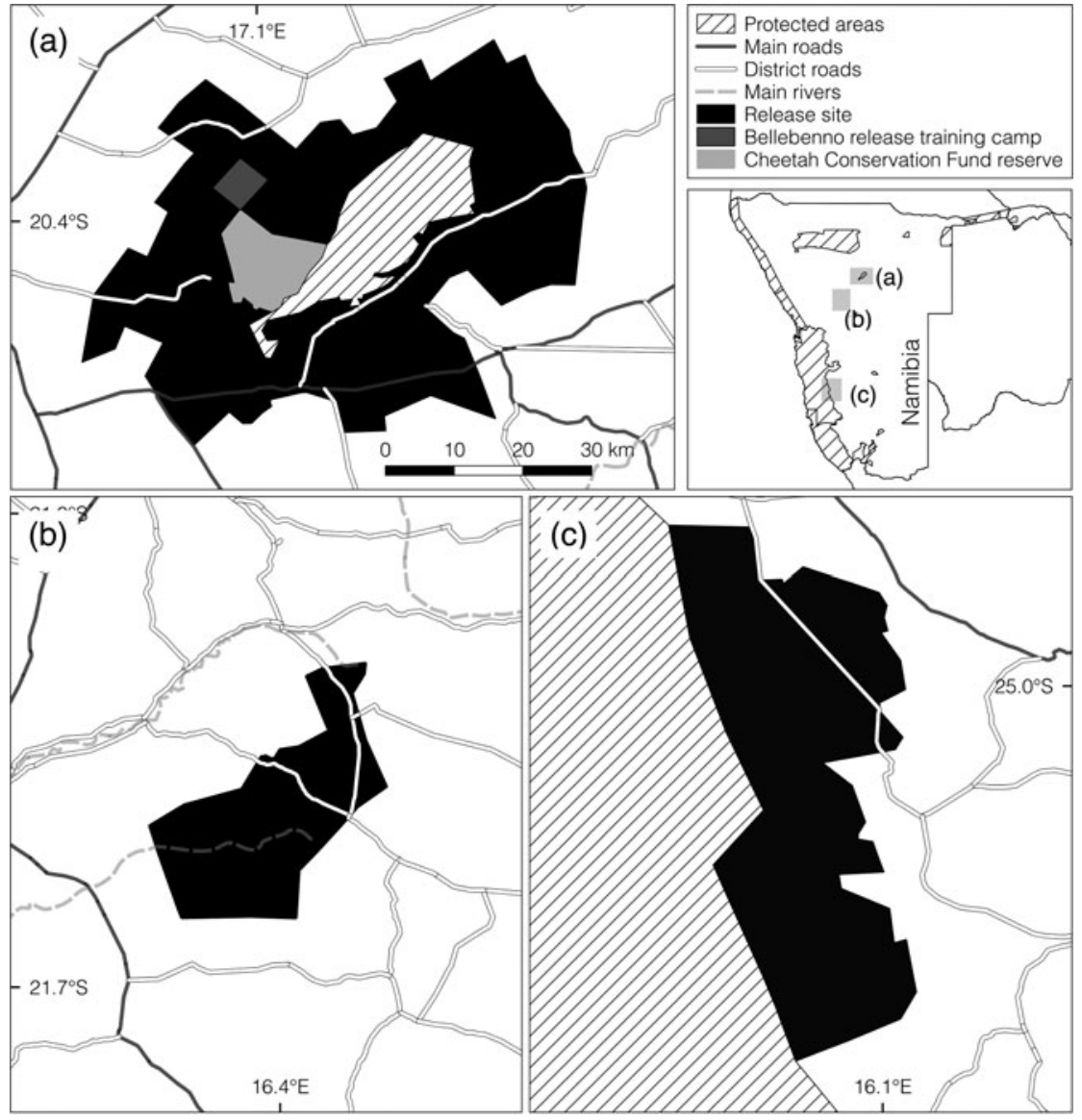

FIG. 1 Locations of the three sites where cheetahs Acinonyx jubatus were released: (a) Greater Waterberg Landscape in north-central Namibia, including the Bellebenno release training camp; (b) Erindi Private Game Reserve in north-central Namibia; and (c) NamibRand Nature Reserve in south-central Namibia. early age, both male and female cheetah cubs can form coalitions with unrelated individuals in captivity. When possible, we bonded release candidates to form artificial coalitions, with the goal of improving each member's survival probability (Hunter, 1998; Gusset et al., 2008; Boast et al., 2018; Marneweck et al., 2019).

Age at release To increase their ability to integrate into their new environment, we only released fully-grown candidates that were at least 2.5 years old.

\section{Pre-release protocol}

Husbandry Release candidates were housed in large offexhibit enclosures and were kept in good physical and mental condition through daily exercise by allowing them to chase the feeding vehicle (see Supplementary Material 1 for additional information).

Release candidate preparation One month prior to a planned release, feeding was changed to a diet consisting 
TABLE 2 Classification criteria for determining habituation level of release candidates. Adapted from Weise et al. (2015).

\begin{tabular}{ll}
\hline $\begin{array}{l}\text { Habituation } \\
\text { level }\end{array}$ & Behavioural criteria \\
\hline Not-habituated & $\begin{array}{l}\text { No tolerance for humans in any context: } \\
\text { direct observation nearly impossible; } \\
\text { extreme levels of stress when confined } \\
\text { Avoids human presence except in feeding } \\
\text { cemi-habituated } \\
\text { after being fed; direct observation possible } \\
\text { from suitable distance \& in certain contexts; } \\
\text { high levels of stress when confined } \\
\text { Tolerates human presence in non-feeding } \\
\text { contexts; does not exhibit normal cheetah } \\
\text { flight response when approached; } \\
\text { low stress levels when confined }\end{array}$ \\
\hline
\end{tabular}

of whole carcasses of natural prey. This provided the release candidates with the experience to open and feed from intact carcasses and prepared their system to handle less frequent, larger quantities of food. A veterinarian registered with the Veterinary Council of Namibia performed a full health examination under anaesthesia on all candidates prior to release to verify good health status and fit cheetahs with a GPS/VHF collar to enable post-release monitoring. All releases were done under permission from Namibia's Ministry of Environment, Forestry and Tourism. For additional details on release preparation and collaring, see Supplementary Material 2.

Release type We used two release types (hard and soft). In a hard release individuals are freed into the new environment immediately upon arrival, whereas in a soft release individuals are allowed a period of acclimation to their new environment in a temporary holding facility (a boma) before release (Hunter et al., 2007). A soft release aims to suppress the tendency to return to the location of capture, known as homing, thereby encouraging the establishment of a home range within the release site (Hayward et al., 2007; Briers-Louw et al., 2019). Cheetahs soft-released in this study were kept in holding bomas for 1-5 months. Any animal released into the Greater Waterberg Landscape from an enclosure located within the landscape was considered soft-released. For some individuals, we used both release types as some cheetahs were released more than once during the study (Table 3). Pre-release hunting training may increase post-release survival (Houser et al., 2011). Therefore, we trialled a release preparation technique, training-release, on some individuals, providing them with hunting experience in a monitored and relatively safe environment (Bellebenno release training camp; Fig. 1) prior to release. Training-release trials took place early in our study and were used for individuals or groups that had characteristics prompting concern for their survival chances (e.g. long periods of time in captivity, physical issues). A given
TABLE 3 Breakdown of releases by release site, release type and released cheetahs.

\begin{tabular}{|c|c|c|}
\hline Release site & $\begin{array}{l}\text { Release } \\
\text { type }\end{array}$ & Cheetahs \\
\hline $\begin{array}{l}\text { Training-release } \\
\text { reserve }\end{array}$ & Training & $\begin{array}{l}\mathrm{SF} 2, \mathrm{SF}^{1}, \mathrm{SF}^{1}, \mathrm{CF} 1^{1}, \mathrm{CF} 2, \\
\mathrm{FC} 1, \mathrm{CM}^{1}, \mathrm{CM} 2\end{array}$ \\
\hline $\begin{array}{l}\text { Greater Waterberg } \\
\text { Landscape }\end{array}$ & Soft & $\begin{array}{l}\text { SF1, SF2 }{ }^{2}, \text { SF }^{2} \text {, SF6, SF7, SF8, } \\
\text { SF9, SF10, SF11, SF12 }\end{array}$ \\
\hline $\begin{array}{l}\text { Erindi Private } \\
\text { Game Reserve }\end{array}$ & $\begin{array}{l}\text { Soft } \\
\text { Hard }\end{array}$ & $\begin{array}{l}\mathrm{SF}^{2}, \mathrm{CM} 4 \\
\text { SF2 }^{2}, \text { FC1, CF2, CM2 }\end{array}$ \\
\hline NamibRand & $\begin{array}{l}\text { Soft } \\
\text { Hard }\end{array}$ & $\mathrm{CM}^{2}, \mathrm{CF} 3$ \\
\hline Nature Reserve & Hard & $\mathrm{CM}^{2}$ \\
\hline
\end{tabular}

${ }^{1}$ Indicates cheetahs that failed their training-release and were returned to captivity; bold font indicates individuals that underwent training-release prior to release.

${ }^{2}$ Indicates cheetahs released more than once.

candidate group or individual was released into the training camp and monitored intensely during daylight hours, facilitated by the manageable size (4,00o ha) of the camp. During these trials cheetahs were fed smaller portions more frequently (every 2-3 days) than during an actual release (Supplementary Material 3). Once an individual or coalition achieved independence in terms of hunting, preparation began for the final release. Individuals that failed to transition towards the expected routine of a wild cheetah were returned to permanent captivity.

\section{Post-release monitoring protocol}

Monitoring routine/schedule We attempted to locate each individual or group twice daily (during 05.00-09.00 and 15.00-19.00) and recorded all actions during the monitoring period. The monitoring team used collar GPS data each morning to find the cheetahs or began tracking from the individual's last known location if it only had a VHF collar. The team determined and prioritized monitoring activity based upon the overnight developments captured by the GPS data or on the events of the previous day. Additionally, as cheetahs rarely scavenge, we visited GPS cluster points to identify carcass remains as evidence for any kill made by the individual or coalition.

Supplemental feeding/water and medical aid Supplemental feeding and watering occurred as necessary with all released cheetahs. If a cheetah failed to make a kill when expected or necessary (based on its body condition), we fed a large meal (5-8 $\mathrm{kg}$ per cheetah of either horse/donkey Equus sp. or game meat) to that individual or group. If an individual or group had still not made a successful kill after the second or third week of release, we started decreasing the frequency of feedings to promote hunting. Minimal feeding frequency was maintained to ensure that cheetahs were not at risk of loss of condition or starvation. Supplemental feeding 
stopped as soon as cheetahs began making successful kills at regular intervals (Supplementary Material 3). A registered veterinarian was on standby for any emergency situation.

End of intensive post-release monitoring period Once a cheetah settled into a behavioural routine expected of a wild cheetah (i.e. no longer requiring regular supplemental feeding, visiting water points, and stabilization of initial exploratory behaviour; Marker et al., 2018a,b; Wachter et al., 2018), the monitoring team would end the intensive post-release monitoring period. From then on, cheetahs were only monitored via their GPS/VHF collars and observed when they were easy to find or when they were seen opportunistically.

\section{Assessment of release success}

We evaluated the success of released cheetahs based on achieved independence and survival.

Independence achieved We considered a released cheetah to have achieved independence once they began making kills at regular intervals and no longer required supplemental feeding for survival. If an individual or group failed to reach independence or displayed behaviour suggesting they would fail to achieve independence, we considered their release a failure and returned them to permanent captivity.

Survival To assess cheetah survival, we calculated KaplanMeier survival estimates with the product limit estimator using a staggered entry design (Kaplan \& Meier, 1958; Pollock et al., 1989). This method allowed the inclusion of animals entering the study at irregular intervals and accommodates data from censored individuals (individuals with unknown fates when collars fail or deplete). Survival estimates were based on the time individuals survived during both the training-release (if applicable) and the post-release monitoring period. We did not include released individuals that returned to captivity after a failed training-release or release in our survival analyses. We used log rank tests to compare survival curves between social grouping (male coalition, reserve; female coalition, reserve; single females, reserve; single females, farmland), and trained/non-trained individuals (Pollock et al., 1989). Two continuous variables were also investigated, after being transformed into categorical factors. Age when orphaned was split into individuals orphaned at $<6$ months of age and individuals orphaned at $\geq 6$ months of age, to assess whether individuals $<6$ months old could be considered for release when bonded to older individuals. Captivity time was divided into individuals in captivity $<1,500$ days and $\geq 1,500$ days. Survival was estimated for consecutive 3-month periods to investigate differences throughout the post-release period, as Weise et al. (2015) detected increased mortality in the first 3 months after the release of translocated cheetahs. Analysis was done in $R$ 4.0.4 (R Core Team, 2021).

\section{Results}

\section{Selected release candidates}

Of 86 wild-born, captive-raised orphaned cheetahs rescued during 2001-2012, $42 \%$ were selected for rehabilitation based on age on arrival and health status. The 36 individuals (15 males, 21 females) were divided into 20 release groups (Table 3); four male coalitions ( $\mathrm{CM}_{1}$ to $\left.\mathrm{CM}_{4}\right)$, three female coalitions ( $\mathrm{CF}_{1}$ to $\mathrm{CF}_{3}$ ), 12 single females ( $\mathrm{SF} 1$ to $\mathrm{SF}_{12}$ ), and one female with cubs (FC1). Candidates selected for release were orphaned at 3-13 months of age (mean 7.28 $\pm 2.61 \mathrm{SD}$ months), spent 1.5-9 years in captivity (mean $4.76 \pm$ SD 1.87 years) before release, and were released at 2.5-10 years of age (mean $5.56 \pm 1.81$ years).

\section{Releases}

Releases took place during 2004-2018. Eight training-releases involving 17 individuals were attempted. Three $\left(\mathrm{CF}_{2}, \mathrm{FC} 1\right.$, $\mathrm{CM}_{2}$ ) of the eight groups were hard-released in Erindi, and a fourth $\left(\mathrm{SF}_{2}\right)$ was soft-released into the Greater Waterberg Landscape prior to being hard-released into Erindi (Table 3). Of the 12 groups that did not undergo training, nine were softreleased into the Greater Waterberg Landscape, one $\left(\mathrm{CF}_{3}\right)$ was soft-released into NamibRand, one $\left(\mathrm{SF}_{5}\right)$ was soft-released twice, first into the Greater Waterberg Landscape and later into Erindi following veterinary treatment for an injury sustained in the first release, and one $\left(\mathrm{CM}_{3}\right)$ was first hardreleased into NamibRand, but recaptured as they began moving out of the Reserve, and then soft-released back into NamibRand (Table 3).

\section{Evaluation of release success}

Training-release outcomes Of the $17(7 \mathrm{M}, 10 \mathrm{~F})$ cheetahs that underwent training-release, $52 \%(\mathrm{n}=9 ; 4 \mathrm{M}, 5 \mathrm{~F})$ successfully reached independence and were subsequently released, and $48 \%(\mathrm{n}=8 ; 4 \mathrm{M}, 4 \mathrm{~F})$ failed to reach independence and were returned to captivity. Of these eight, two females $\left(\mathrm{CF}_{1}\right)$ escaped the training camp and killed a goat, two females $\left(\mathrm{SF}_{3}, \mathrm{SF}_{4}\right)$ failed to make successful kills and throughout their training-release period showed abnormally low interest in trying to hunt, and four males ( $\mathrm{CM} 1)$ remained at the release enclosure and did not explore the training camp or attempt to hunt.

Time to independence Of the 36 cheetahs selected for release, $75 \%$ (10 M, 17 F) achieved independence. Eight individuals failed to achieve independence during the training- 
release, and one male (\#NAAJU1616 of $\mathrm{CM}_{4}$ ) was killed by a leopard Panthera pardus before the coalition had achieved independence. Considering only cheetahs that underwent final release, independence was achieved by $96 \%$ of released individuals. Each individual or group achieving independence was supplemental fed on average 17 times (median 7 feedings, range 1-112 feedings) and made its first known kill after 18 days (median 7 days, range 3-113 days). FC1 struggled post-release, probably because of her dependent cubs, and as a result was fed more frequently (112 feedings) to ensure that the cubs were properly nourished. Most individuals or groups ( 25 cheetahs) achieved independence within 2 weeks after release and only required 1-3 supplemental feedings before making their first kills.

Survival The eight individuals that failed their training-release trials were not included in the survival analyses, reducing the total number of individuals used for analysis to $28(11 \mathrm{M}, 17$ F). Of these, $68 \%(6 \mathrm{M}, 13 \mathrm{~F})$ were recorded dead at the time of analysis, and the fate of $32 \%(5 \mathrm{M}, 4 \mathrm{~F})$ was unknown because of collar failure or depleted batteries. The annual KaplanMeier survival estimate for all released individuals was 0.61 (95\% CI 0.45-0.84; Fig. 2, Table 4). Social grouping did not significantly affect survival, but males in coalitions tended to live longer compared to females in coalitions and single females. The six deaths recorded on farmland were attributed to both anthropogenic (34\%) and natural causes (64\%). Of the four cheetahs that died of natural causes, two were from hunting injuries and two were killed by leopards. For the 13 recorded deaths in a reserve, $69 \%$ were attributed to natural causes and $31 \%$ to unknown causes. Of the nine cheetahs that died of natural causes, two died of old age, two of hunting injuries, one was killed by baboons Papio ursinus and four by competing predators (two leopard, one lion Panthera leo, one spotted hyaena Crocuta crocuta). No differences in survival were found for the variables age when orphaned, time spent in captivity, and training-release (Fig. 2, Table 4). No deaths occurred as a result of starvation, suggesting that cheetahs, with strategic support provided by post-release monitoring, can acquire the skills required to support themselves despite their history. Overall survival estimates at 3, 6, 9 and 12 months remained stable, without any increased mortality in the first 3 months post-release (Fig. 3). Survival in the first and second years did not differ significantly $\left(\chi^{2}=0, \mathrm{df}=1, \mathrm{P}=0.8\right)$. The increase in survivorship after 15 months is a result of five individuals (18\%) surviving beyond 2 years-post release.

Reproductive success Successful reproduction is the ultimate measure of the success of release efforts. Although our data is insufficient for statistical analyses, the following observations were made: SF5, SF6, SF10 and SF11 mated with and were impregnated by wild males. $\mathrm{SF}_{5}$ raised two female cubs to independence, SF1o gave birth to a litter of cubs before her collar failed. During the necropsy on SF6, five unborn cubs were found. SF11 gave birth to two litters, lost the first but raised the second to 11 months of age before being killed by a leopard. $\mathrm{CM}_{4}$ were seen on several occasions with female cheetahs, and were observed mating with a wild female.

\section{Discussion}

Following our release protocol, the success rate of animals achieving independence was high. Furthermore, annual postrelease survival rate was similar to other cheetahs translocated into a free-ranging reserve (NamibRand) in Namibia (annual Kaplan-Meier survival estimate 0.57, 95\% CI 0.35-0.76; Weise et al., 2015) and mortality was comparable to cheetahs in a fenced reserve in South Africa (Bissett \& Bernard, 2011). Independence was achieved quickly for most individuals $(<2$ weeks $)$ and we had evidence of successful reproduction events for some released individuals.

Neither age when orphaned nor time in captivity affected the survival probability of released cheetahs. Therefore, our protocol for pre-release management to achieve release candidate status for individuals orphaned younger than 6 months through bonding them to candidates orphaned at an older age seems effective. These findings are contrary to those of previous studies in which time in captivity had significant negative influence over survival, as they show success in releasing captive-raised individuals (Jule et al., 2008; Weise et al., 2015; Boast et al., 2018).

Although the training-releases did not significantly improve survival, one advantage they may offer is the opportunity to screen candidates that meet requirements but that would benefit from additional assessment to confirm their ability to reach independence (Gusset et al., 2006; Houser et al., 2011). However, we suggest caution when employing such a technique to provide pre-release hunting training to large carnivores. As natural predator-prey interactions are difficult to recreate, the behaviour and strategy developed within a training environment may not be adequate for a final release. With intensive post-release monitoring according to our protocol, an animal can be reliably supported with supplemental feeding/water and other interventions (see Methods) as necessary until it has reached independence. Thus, we suggest that intensive post-release monitoring within the release site provides the animal with the safest and most stable conditions for acquiring the necessary hunting and survival skills relevant to that release site.

Past studies of carnivore releases have demonstrated the challenges associated with homing behaviour (Linnell et al., 1997; Yiu et al., 2015), which has also been observed in captive-raised and released animals of other species (Henshaw \& Stephenson, 1974; Ridgway \& Robison, 1985). Although we could not test for differences between soft and hard release, we recommend implementing soft releases 

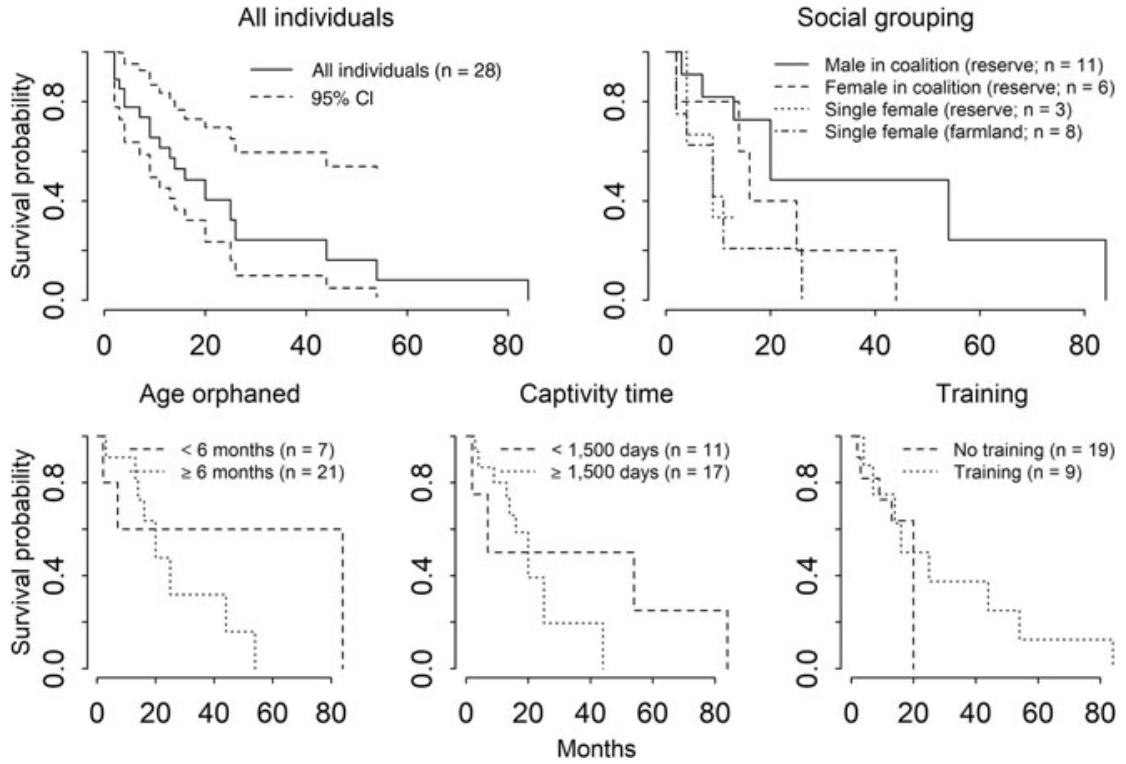

FIG. 2 Kaplan-Meier survivorship curves for all the released captive-reared cheetahs and comparisons for the variables social grouping, age when orphaned, captivity time and training-release.

TABLE 4 Annual Kaplan-Meier (KM) survivorship estimates with 95\% confidence intervals of released captive-reared cheetahs. $\chi^{2}$ values, degrees of freedom and probability are reported for the log rank test comparing survivorship for different variables.

\begin{tabular}{|c|c|c|c|c|c|}
\hline Variable & Treatment & Annual KM survivorship (95\% CI) & $\chi^{2}$ & df & $\mathrm{P}$ \\
\hline \multirow[t]{4}{*}{ Social grouping } & Male in coalition (reserve) & $0.82(0.62-1.00)$ & 6.5 & 3 & 0.1 \\
\hline & Female in coalition (reserve) & $0.80(0.52-1.00)$ & & & \\
\hline & Single female (reserve) & $0.33(0.07-1.00)$ & & & \\
\hline & Single female (farmland) & $0.21(0.04-1.00)$ & & & \\
\hline \multirow[t]{2}{*}{ Age when orphaned } & $<6$ months & $0.50(0.23-1.00)$ & 0.5 & 1 & 0.5 \\
\hline & $\geq 6$ months & $0.65(0.47-0.90)$ & & & \\
\hline \multirow{2}{*}{ Captivity time } & $<1,500$ days & $0.45(0.21-0.96)$ & 0 & 1 & 1 \\
\hline & $\geq 1,500$ days & $0.71(0.52-0.96)$ & & & \\
\hline \multirow{2}{*}{ Training } & No & $0.55(0.36-0.84)$ & 1 & 1 & 0.3 \\
\hline & Yes & $0.75(0.50-1.00)$ & & & \\
\hline All individuals & & $0.61(0.45-0.84)$ & & & \\
\hline
\end{tabular}

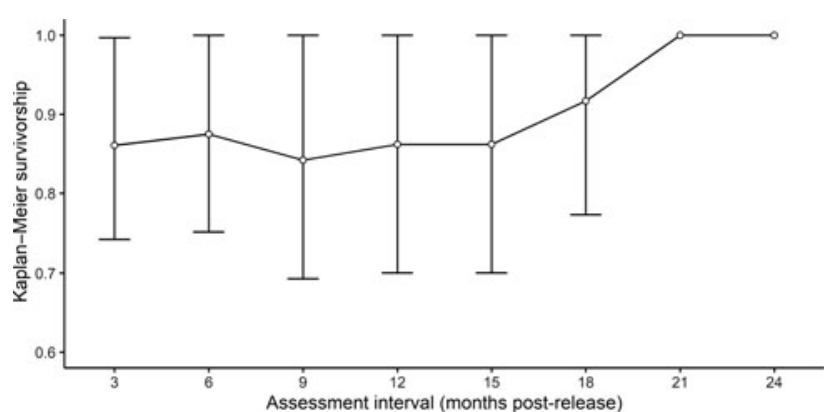

FIG. 3 Three-month interval Kaplan-Meier survival analysis for released cheetahs. Error bars show 95\% CI of the Kaplan-Meier estimate.

based upon past findings and our experience with $\mathrm{CM}_{4}$. $\mathrm{CM}_{4}$ only established themselves after a soft release following a hard release that failed because of extensive exploratory roaming.
Although our results did not show significantly higher survival for releases into reserves, reserves generally exclude the risk of conflict with humans. Additionally, farmland restricted the capacity of our team to monitor and assist released cheetahs. If a cheetah crossed a farm border onto a property where we failed to obtain permission for monitoring, that individual ceased to be monitored until it moved onto a property where it could be monitored. Our inability to closely monitor some released cheetahs moving across property borders perhaps influenced survival probability, as we were unable to provide these individuals with as much support (e.g. supplemental feeding, veterinary assistance) as individuals released into a reserve, where monitoring ability is more secure.

The annual survival estimate obtained for cheetahs released into large or fenced reserves was similar to the annual survival estimate of 0.85 for cheetahs translocated into fenced reserves in South Africa (Marnewick et al., 2009). Buk et al. (2018) found predators to be responsible for 
53.2\% of known deaths for cheetahs translocated into fenced reserves in South Africa. Competing predators were responsible for $44.4 \%$ ( 4 cheetahs) of the deaths with known causes for cheetahs released into reserves in our study, suggesting that predator naivety is not more of a concern for rehabilitated cheetahs than for their wild counterparts. Unfortunately, reserves are often isolated and individuals are not able to contribute to the free-ranging gene pool unless intensive metapopulation management is employed to create gene flow between these isolated populations (Marnewick et al., 2009; Boast et al., 2018). Our findings will be applicable to future reintroduction efforts into reserves within the cheetah's historical range after problems that caused extirpation have been addressed (IUCN/SSC, 2013).

Released coalitions tended to have a higher estimated survivorship than single individuals, which may be attributable to increased chances of obtaining core territories. For male cheetahs these are smaller sections of overall home ranges that reliably provide food, shelter and water for its holder (Caro, 1994; Melzheimer et al., 2018). Moreover, living in coalitions may improve hunting success and facilitate defence against other cheetah coalitions (Caro, 1994; Durant et al., 2004). Our small sample did not allow us to test for differences in survival between sexes, but males tended to live longer than females. Longevity of females is particularly important as they need a minimum of 2 years post-release to successfully raise offspring (Laurenson, 1993). Males on the other hand may contribute their genes relatively quickly and may therefore be of higher conservation benefit in the short-term. Paying close attention to females during the post-release monitoring period is critical for maximizing the success of the release of females.

Although releases are time-intensive and costly, permanent captivity is also expensive (c. USD 5,000 annually per animal). Therefore releasing a captive-raised individual presents financial benefits in addition to the biological benefit of returning an individual to the wild gene pool. Thus, we argue that the benefits of releasing captive-raised individuals probably outweigh concerns regarding its cost (Weise et al., 2014; Boast et al., 2018) and, with appropriate planning, such releases could provide genetic benefits to wild populations. Our study challenges recent critique that using captive-raised large carnivores for release presents little value for conservation (Hunter \& Rabinowitz, 2009; Weise et al., 2015).

By carefully selecting release candidates, artificially forming coalitions where possible, balancing habituation levels during captivity, and providing strategic support during post-release monitoring, we have demonstrated that captive-reared cheetahs orphaned at age of 6 months or older can transition fully back into the wild. Moreover, cheetahs orphaned at a younger age can successfully be released if bonded to qualifying individuals before release; this strategy may prove a valuable tool for the development of procedures to release captive-bred individuals successfully. The effectiveness and necessity of post-release monitoring and management is further highlighted by the absence of elevated mortality during the first 3 months post-release (Weise et al., 2015). Our monitoring team always consisted of at least one person with extensive experience working directly with cheetahs. This experience enabled the team to adapt dynamically and respond to the developments of each release, thus improving the survival probability of released cheetahs. Having a veterinarian on standby allowed the team to treat potentially life-threatening injuries, providing the individual with an additional chance to reach independence. Further investigation into the causes of deaths of rehabilitated cheetahs is warranted, to facilitate experimentation with potential mitigation measures. Improved understanding of the specific risks associated with a given release site and the development of post-release management actions to mitigate those risks would improve survival of released individuals.

Although previous dismissal of releasing captive-raised large carnivores is understandable considering past failed release attempts, we argue this conclusion is premature, as most studies have approached the release of captivereared cheetahs with the same pre- and post-release treatment as for wild conspecifics (Jule et al., 2008; Hunter \& Rabinowitz, 2009; Weise et al., 2015; Boast et al., 2018). Such an approach ignores the fact that captive-reared cheetahs lack much of the learning and experience provided by their mother during dependency. Although a lot of survival skills are probably instinctual, they do require trial and error until being fully acquired; by appropriately accounting for this challenge during pre- and post-release management we have shown that orphaned cheetahs are capable of transitioning back into the wild. We recommend that any future release efforts of captive-raised large carnivores be based on design strategies that fully consider the experiential knowledge gaps such individuals possess. Despite the small sample, our study indicates that cheetahs rehabilitated, released and monitored according to our protocol have chances of survival similar to those of wild cheetahs and therefore this technique is a valuable tool for cheetah conservation.

Acknowledgements We thank Namibia's Ministry of Environment, Forestry and Tourism for supporting this research, all Cheetah Conservation Fund supporters that made this study financially feasible, all Cheetah Conservation Fund staff and volunteers who contributed to this research, Lorraine Boast for her comments on the text, and all the partners and release sites (the Greater Waterberg Landscape, Erindi Private Game Reserve, and NamibRand Nature Reserve), who worked with us to make this research possible.

Author contributions Study design: EHW, LM, AS-K; fieldwork: EHW, LM, SV; writing, data analysis, revision: all authors.

\section{Conflicts of interest None.}


Ethical standards This research adhered to IUCN definitions and guidelines for reintroduction and translocations and to the Guidelines for Ethical Treatment of Animals in Applied Animal Behaviour and Welfare Research standards of the International Society for Applied Ethology, and otherwise abided by the Oryx ethical standards. All research and work with animals was approved by Namibia's Ministry of Environment, Forestry and Tourism.

\section{References}

Bissett, C. \& Bernard, R.T.F. (2011) Demography of cheetahs in fenced reserves in South Africa: implications for conservation. African Journal of Wildife Research, 41, 181-191.

Boast, L.K., Chelysheva, E.V., van der Merwe, V., Schmidt-Küntzel, A., Walker, E.H., Cilliers, D. et al. (2018) Cheetah translocation and reintroduction programs: past, present, and future. In Cheetahs: Biology and Conservation: Biodiversity of the World: Conservation from Genes to Landscapes (eds P. Nyhus, L. Marker, L. Boast \& A. Schmidt-Küntzel), pp. 275-289. Academic Press, London, UK.

Briers-Louw, W.D., Verschueren, S. \& Leslie, A.J. (2019) Big cats return to Majete Wildlife Reserve, Malawi: evaluating reintroduction success. African Journal of Wildlife Research, $49,34-50$

Broekhuis, F., Cozzi, G., Valeix, M., Mcnutt, J.W. \& Macdonald, D.W. (2013) Risk avoidance in sympatric large carnivores: reactive or predictive? Journal of Animal Ecology, 82, 1098-1105.

Buk, K.G., van der Merwe, V.C., Marnewick, K. \& Funston, P.J. (2018) Conservation of severely fragmented populations: lessons from the transformation of uncoordinated reintroductions of cheetahs (Acinonyx jubatus) into a managed metapopulation with self-sustained growth. Biodiversity and Conservation, 27, 3393-3423.

CARo, T.M. (1994) Cheetahs of the Serengeti Plains: Group Living in an Asocial Species. University of Chicago Press, Chicago, USA.

Durant, S., Mitchell, N., Ipavec, A. \& Groom, R. (2015) Acinonyx jubatus. In IUCN Red List of Threatened Species 2015. dx.doi.org/10. 2305/IUCN.UK.2015-4.RLTS.T219A50649567.en [accessed 25 June 2021].

Durant, S.M. (1998) Competition refuges and coexistence: an example from Serengeti carnivores. Journal of Animal Ecology, $67,370-386$

Durant, S.M. (200o) Predator avoidance, breeding experience and reproductive success in Endangered cheetah, Acinonyx jubatus. Animal Behaviour, 60, 121-130.

Durant, S.M., Kelly, M. \& Caro, T.M. (2004) Factors affecting life and death in Serengeti cheetahs: environment, age, and sociality. Behavioral Ecology, 15, 11-22.

Gusset, M., Ryan, S.J., Hofmeyr, M., van Dyk, G., DaviesMostert, H.T., Graf, J.A. et al. (2008) Efforts going to the dogs? Evaluating attempts to re-introduce Endangered wild dogs in South Africa. Journal of Applied Ecology, 45, 100-108.

Gusset, M., Slotow, R. \& Somers, M.J. (2006) Divided we fail: the importance of social integration for the re-introduction of endangered African wild dogs (Lycaon pictus). Journal of Zoology, 270, 502-511.

Hayward, M.W., Adendorff, J., O’Brien, J., Sholto-Douglas, A., Bissett, C., Moolman, L.C. et al. (2007) Practical considerations for the reintroduction of large, terrestrial, mammalian predators based on reintroductions to South Africa's Eastern Cape Province. The Open Conservation Biology Journal, $1,1-11$
Henshaw, R.E. \& Stephenson, R.O. (1974) Homing in the gray wolf (Canis lupus). Journal of Mammalogy, 55, 234-237.

Houser, A., Gusset, M., Bragg, C.J., Boast, L.K. \& Somers, M.J. (2011) Pre-release hunting training and post-release monitoring are key components in the rehabilitation of orphaned large felids. South African Journal of Wildlife Research, 41, 11-20.

HUnter, L. (1998) Early post-release movements and behaviour of reintroduced cheetahs and lions, and technical considerations in large carnivore restoration. In Proceedings of a Symposium on Cheetahs as Game Ranch Animals (ed. B.L. Penzhorn), pp. 72-82. South African Veterinarian Association, Onderstepoort, South Africa.

Hunter, L.T.B., Pretorius, K., Carlisle, L.C., Rickelton, M., Walker, C., Slotow, R. \& Skinner, J.D. (2007) Restoring lions Panthera leo to northern KwaZulu-Natal, South Africa: short-term biological and technical success but equivocal long-term conservation. Oryx, 41, 196-204.

Hunter, L.T.B. \& Rabinowitz, A. (2009) Felid reintroduction using captive founders: poor science and worst practices. Cat News, $51,28-29$.

IUCN/SSC (2013) Guidelines for Reintroductions and Other Conservation Translocations. IUCN Species Survival Commission, Gland, Switzerland.

Jule, K.R., Leaver, L.A. \& Lea, S.E.G. (2008) The effects of captive experience on reintroduction survival in carnivores: a review and analysis. Biological Conservation, 141, 355-363.

Kaplan, E.L. \& Meier, P. (1958) Nonparametric estimation from incomplete observations. Journal of the American Statistical Association, 53, 457-481.

Laurenson, M.K. (1993) Early maternal behavior of wild cheetahs: implications for captive husbandry. Zoo Biology, 12, 31-43.

Lindsey, P., Tambling, C.J., Brummer, R., Davies-Mostert, H., Hayward, M., Marnewick, K. \& Parker, D. (2011) Minimum prey and area requirements of the Vulnerable cheetah Acinonyx jubatus: implications for reintroduction and management of the species in South Africa. Oryx, 45, 587-599.

Linnell, J.D.C., Aanes, R., Swenson, J.E., Odden, J. \& Smith, M.E. (1997) Translocation of carnivores as a method for managing problem animals: a review. Biodiversity \& Conservation, 6, 1245-1257.

Marker, L., Cristescu, B., Dickman, A., NGhikembua, M.T., BoAst, L.K., Morrison, T. et al. (2018a) Ecology of free-ranging cheetahs. In Cheetahs: Biology and Conservation (eds P. Nyhus, L. Marker, L. Boast \& A. Schmidt-Küntzel), pp. 107-119. Academic Press, London, UK.

Marker, L., Cristescu, B., Morrison, T., Flyman, M.V., Horgan, J., Sogbohossou, E.A. et al. (2018b) Cheetah rangewide status and distribution. In Cheetahs: Biology and Conservation (eds P. Nyhus, L. Marker, L. Boast \& A. Schmidt-Küntzel), pp. 33-54. Academic Press, London, UK.

Marker, L., Dickman, A., Wilkinson, C., Schumann, B. \& Fabiano, E. (2007) The Namibian cheetah: status report. Cat News, Special Issue 3, 4-13. IUCN/Species Survival Commission Cat Specialist Group, Bern, Switzerland.

Marker-Kraus, L., Kraus, D., Hurlbutt, S. \& Barnett, D. (1996) Cheetah Survival on Namibian Farmlands. Cheetah Conservation Fund, Windhoek, Namibia.

Marneweck, C., Becker, P.A., Beverley, G., Davies-Mostert, H.T., DU Plessis, C., Forssman, K. et al. (2019) Factors affecting the success of artificial pack formation in an endangered, social carnivore: the African wild dog. Animal Conservation, 22, 493-502.

Marnewick, K., Hayward, M.W., Cilliers, D. \& Somers, M.J. (2009) Survival of cheetahs relocated from ranchland to fenced. In Reintroduction of Top-Order Predators (eds M.W. Hayward \& M.J. Somers), pp. 282-306. Blackwell Publishing, Oxford, UK. 
Melzheimer, J., Streif, S., Wasiolka, B., Fischer, M., Thalwitzer, S., Heinrich, S.K. et al. (2018) Queuing, takeovers, and becoming a fat cat: long-term data reveal two distinct male spatial tactics at different life-history stages in Namibian cheetahs. Ecosphere, 9, e02308.

Morsbach, D. (1987) Cheetah in Namibia. Cat News, 6, 25-26.

Pollock, K.H., Winterstein, S.R., Bunck, C.M. \& Curtis, P.D. (1989) Survival analysis in telemetry studies: the staggered entry design. The Journal of Wildlife Management, 53, 7-15.

R Core Team (2021) R: A Language and Environment for Statistical Computing. R Foundation for Statistical Computing, Vienna, Austria.

Ridgway, S.H. \& Robison, C.C. (1985) Homing by released captive California sea lions, Zalophus californianus, following release on distant islands. Canadian Journal of Zoology, 63, 2162-2164.

Schwartz, K.R., Gusset, M., Crosier, A.E., Versteege, L., Eyre, S., Tiffin, A. \& Kotzé, A. (2018) The role of zoos in cheetah conservation: integrating Ex situ and In situ conservation action. In Cheetahs: Biology and Conservation (eds P. Nyhus, L. Marker, L. Boast \& A. Schmidt-Küntzel), pp. 323-334. Academic Press, London, UK.

SNyder, N.F.R., Derrickson, S.R., Beissinger, S.R., Wiley, J.W., Smith, T.B., Toone, W.D. \& Miller, B. (1996) Limitations of captive breeding in endangered species recovery. Conservation Biology, 10, 338-348.

Swanson, A., Arnold, T., Kosmala, M., Forester, J. \& Packer, C. (2016) In the absence of a 'landscape of fear': how lions, hyenas, and cheetahs coexist. Ecology and Evolution, 6, 8534-8545.

Swanson, A., Caro, T., Davies-mostert, H., Mills, M.G.L., Macdonald, D.W., Borner, M. et al. (2014) Cheetahs and wild dogs show contrasting patterns of suppression by lions. Journal of Animal Ecology, 83, 1418-1427.

Taylor, G., Canessa, S., Clarke, R.H., Ingwersen, D., Armstrong, D.P., Seddon, P.J. \& Ewen, J.G. (2017)
Is reintroduction biology an effective applied science? Trends in Ecology and Evolution, 32, 873-880.

Vebber, L., Noack, J., Heyns, L., Rodenwoldt, D. \& Edwards, S. (2020) Rehabilitated cheetahs exhibit similar prey selection behaviour to their wild counterparts: a case study of prey selection by rehabilitated cheetah released into an enclosed reserve in north-central Namibia. Namibian Journal of Environment, 4, 13-19.

VICKERY, S.S. \& MASON, G.J. (2003) Behavioural persistence in captive bears and its implication for reintroduction. Ursus, 14, 35-43.

Wachter, B., Broekhuis, F., Melzheimer, J., Horgan, J., Chelysheva, E.V., Marker, L. et al. (2018) Behavior and communication of free-ranging cheetahs. In Cheetahs: Biology and Conservation (eds P. Nyhus, L. Marker, L. Boast \& A. Schmidt-Küntzel), pp. 121-134. Academic Press, London, UK.

Weilenmann, M., Gusset, M., Mills, D. R., Gabanapelo, T. \& Schiess-Meier, M. (2010) Is translocation of stock-raiding leopards into a protected area with resident conspecifics an effective management tool? Wildlife Research, 37, 702-707.

Weise, F.J., Lemeris, J., Bowden, A., Venter, C., van Vuuren, M. \& van VuUren, R.J. (2015) Cheetahs (Acinonyx jubatus) running the gauntlet: an evaluation of translocations into free-range environments in Namibia. PeerJ, 3, e1346.

Weise, F.J., Stratford, K.J. \& van Vuuren, R.J. (2014) Financial costs of large carnivore translocations-accounting for conservation. PLOS ONE, 9, e105042.

Wilson, A.C. \& Price, M.R.S. (1994) Reintroduction as a reason for captive breeding. In Creative Conservation (eds P.J.S. Olney, G.M. Mace \& A.T.C. Feistner), pp. 243-264. Springer Netherlands, Dordrecht, The Netherlands.

Yiu, S.W., Keith, M., Karczmarski, L. \& Parrini, F. (2015) Early post-release movement of reintroduced lions (Panthera leo) in Dinokeng Game Reserve, Gauteng, South Africa. European Journal of Wildlife Research, 61, 861-870. 